\title{
Performance Analysis of Adaptive Scheduling in Integrated Services UMTS Networks
}

\author{
Remco Litjens ${ }^{\star}$ and Hans van den Berg ${ }^{\star, \dagger}$ \\ ${ }^{\star}$ Knowledge Innovation Center, KPN Research, The Netherlands. \{R.Litjens, J.L.vandenBerg\}@kpn.com \\ ${ }^{\dagger}$ Faculty of Mathematical Sciences, University of Twente, The Netherlands, J.L.vandenBerg@math.utwente.nl
}

\begin{abstract}
For an integrated services UMTS network serving speech and data calls, we propose, evaluate and compare different scheduling schemes, which dynamically adapt the shared data transport channel rates to the varying speech traffic load. Within each cell, the assigned data transfer resources are distributed over the present data flows according to certain fairness objectives. The performance of the adaptive schemes is numerically evaluated by means of analytical performance optimisation methods in combination with Monte Carlo simulations.
\end{abstract}

Keywords-UMTS, HSDPA, integrated services networks, adaptive scheduling, Quality of Service, performance evaluation.

\section{INTRODUCTION}

The third-generation Universal Mobile Telecommunications system (UMTS) [5] is designed to support a wide variety of services, e.g. speech and video telephony, file transfer and www browsing, with distinct Quality of service (QOS) requirements.

A number of technological improvements of the initial UMTS system release is standardised under the denominator Highspeed Downlink Packet Access (HSDPA) [8], whose objective it is to enhance the data transfer capabilities and enable the support of downlink peak rates in the range of $8-10 \mathrm{Mbits} / \mathrm{s}$ for best effort packet data services. To this end, HSDPA upgrades UMTS's Downlink shared CHannel (DSCH) to a High Speed DSCH (HS$\mathrm{DSCH}$ ), which supports a variety of enhanced technologies, including higher order modulation combined with fast link adaptation, and fast scheduling centered at the Base Transceiver Station (BTS) with a proposed smaller transmission time interval, in order to reduce delays and facilitate better tracking of the channel variations. The viability of fast link adaptation allows parameterisation of the HS-DSCH by an assigned transmission power [8], where the experienced data rate depends on the radio link quality of the served data flow. An important advantage is the reduced variability of the exerted power levels, since the path gain differences of the served data calls can be dealt with in the time rather than the power domain.

Operational in different forms at different timescales, adaptive scheduling on HS-DSCHS is foreseen to offer significant QOS enhancements for data services. At a timescale of milliseconds, $C / I$-based scheduling exploits the variability of the radio link due to fast fading by multiplexing the data flows in accordance with their instantaneous $C / I$. This optimises the system throughput but may result in a significant degree of unfairness among data flows $[2,6,7]$.

At a timescale of seconds, the appropriate up- and downgrading of the data transport channels in an integrated services UMTS network is another form of adaptive scheduling which exploits both the traffic load variability of a prioritised e.g. speech service and the delay tolerance of data services. The objective of the present paper is to assess the performance gain that can be achieved by the latter type of adaptive scheduling, combining analytical optimisation techniques with Monte Carlo simulations. Scarce earlier related work includes $[3,9]$, where a load control scheme is assessed which (de)activates the dedicated transport channels conveying speech traffic in support of the varying data traffic load, and [9], which investigates the effectiveness of decreasing data rates to reduce interference levels as a form of congestion control. While $[3,9]$ concentrate on heuristic and rather myopic schemes, the adaptive schemes considered here aim to optimise performance in a network-wide sense.

The outline of the paper is as follows. Section II sets the framework for the performance evaluation of the different scheduling schemes which are specified in Section III. The performance evaluation method is outlined in Section IV. Section $V$ presents a numerical study and discusses the results. Section VI ends the main body of this paper with some concluding remarks.

\section{NETWORK AND TRAFFIC MODEL}

We consider the downlink in a network consisting of a set $\mathbb{B}$ of omnidirectional UMTS BTSs each serving a cell with a radius of $R \mathrm{~km}$. Denote by $p_{\max }$ each BTs's transmission power budget and let $\nu$ be the spatially uniform thermal noise level. A singlepath radio propagation model is used with path loss exponent $\varsigma$ and correlated lognormal shadowing [10] with a standard deviation of $\sigma \mathrm{dB}$. The downlink orthogonality factor is denoted $\omega$. The system chip rate is fixed at $R_{\text {chip }}$ Mchips/s.

The considered UMTS network integrates speech and data services. Speech calls are handled on Dedicated transport CHannels (DCHs) with a fixed information bit rate of $R_{\text {speech }} \mathrm{kbits} / \mathrm{s}$, and require a carrier-to-interference ratio no less than $\gamma_{\text {speech }} \equiv$ 
$\left(R_{\text {speech }} / R_{\text {chip }}\right) 10^{\zeta_{\text {spech }} / 10}$ with $\zeta_{\text {speech }}$ (in $\left.\mathrm{dB}\right)$ the speech service's target. Data transfers are multiplexed on HS-DSCHs (one per BTS). Utilising HSDPA's link adaptation feature, the data transfer rate experienced by a given data flow is proportional to its actual carrier-to-interference ratio $\gamma_{\text {data }}$ :

$$
R_{\text {data }}=\frac{R_{\text {chip }}}{10_{\text {data }} / 10} \gamma_{\text {data }}
$$

given an $E_{b} / N_{o}$ target of $\zeta_{\text {data }}$ (in $\mathrm{dB}$ ). The location of calls of either service type is uniformly sampled over the network.

\section{SCHEDULING SCHEMES}

The scheduling schemes consist of a macro- and a microcomponent. The macro-scheduling scheme dimensions the HSDSCHs in accordance with the actual traffic conditions and is parametrised by $p_{\mathrm{HS}-\mathrm{DSCH}}^{\min }$. If $\varphi_{b}$ denotes the fraction of data flows that is served by BTS $b$, then the HS-DSCH transmission power assigned to a BTS $b$ serving at least a single data flow is equal to

$$
p_{\mathrm{HS}-\mathrm{DSCH}}^{b}=p_{\mathrm{HS}-\mathrm{DSCH}}^{\min }+\varphi_{b} \vartheta_{\mathrm{HS}-\mathrm{DSCl}},
$$

$b \in \mathbb{B}$, where the aggregate adaptive HS-DSCH transmission power component $\vartheta_{\mathrm{HS}-\mathrm{DSCH}}$ is to be maximised under the condition that no speech calls are caused to violate their $C / I$ requirements. While $p_{\mathrm{HS} \cdot \mathrm{DSCH}}^{\min }$ is a minimum per-BTS resource assignment, the incorporation of the $\varphi_{b}$ 's establishes a fair sharing of the scheme's adaptivity gain over the data calls. The special case of $p_{\mathrm{HS}-\mathrm{DSCH}}^{\min }=0$ specifies a purely adaptive scheme, while on the other hand, prefixing $\vartheta_{\text {HS-DSCH }}$ at 0 specifies a purely fixed HSDSCH power assignment, which is used for reference purposes.

At each BTS, either a Round Robin (RR) or weighted Round Robin (WRR) micro-scheduling scheme is selected to multiplex the present data flows over the HS-DSCH as dimensioned at the macro-level. Whereas RR scheduling distributes the BTSs' HS$\mathrm{DSCH}$ resources fairly over the different data flows, the WRR scheme establishes equal throughputs within each cell, by appropriately specifying scheduling weights based on the data flows' (average) path gains. Denote by $\phi_{m}^{b}$ the scheduling weight of data flow $m$ served by BTS $b$ such that $\sum_{m: b_{m}=b} \phi_{m}^{b}=1, b \in \mathbb{B}$.

Figure 1 illustrates the different scheduling timescales. At each adaptive scheduling instant, advocated to correspond with significant changes in the traffic loads, the macro- and microscheduling parameters are re-determined. At the fixed heartbeat determined by the HSDPA transmission time intervals, the data flows are then multiplexed according to their current microscheduling weights.

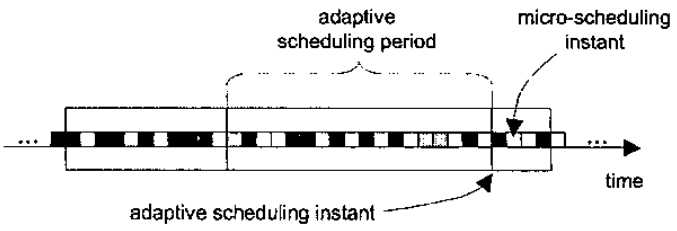

Fig. 1. Scheduling timescales.

\section{Performance eValuation}

The relative performance of the presented scheduling schemes is evaluated by means of analytical optimisation in combination with Monte Carlo simulations. The speech performance is expressed in terms of the outage probability $\mathbf{P}_{s}$, defined as the probability that an arbitrary speech call fails to meet its $C / I$ target, while the data performance is measured by the expected throughput $\mathbf{R}_{d}$ per data flow. Additionally, the conditional speech $\left(\mathbf{P}_{s}(\Omega)\right)$ and data $\left(\mathbf{R}_{d}(\Omega)\right)$ performance are determined as a function of a call's geometry $\Omega$, given by

$$
\Omega_{\mathrm{MS}} \equiv \frac{p_{\max } \mathcal{G}_{\mathrm{MS}} \mathrm{b}_{\mathrm{Ms}}}{\sum_{\Delta \neq b_{\mathrm{MS}}} p_{\max } \mathcal{G}_{\mathrm{Ms}}+\nu},
$$

where $\mathcal{G}_{\mathrm{MS} b}$ denotes the path gain of call MS w.r.t. BTS $b$.

At a given scheduling instant, the constellation of speech and data calls is specified by the set $\mathbb{M}_{s} \equiv\left\{1, \cdots, M_{s}\right\}\left(\mathbb{M}_{d} \equiv\right.$ $\left\{1, \cdots, M_{d}\right\}$ ) of speech (data) calls, the path gains $\mathcal{G}_{m b}, m \in$ $\mathbb{M}_{s} \cup \mathbb{M}_{d}, b \in \mathbb{B}$, and the serving BTS $b_{m}$ assigned to each call $m \in \mathbb{M}$ (based on a 'maximum path gain' criterion). Let $\mathbb{M}_{s}^{b} \equiv$ $\left\{m \in \mathbb{M}_{s}: b_{m}=b\right\}$ and $\mathbb{M}_{d}^{b} \equiv\left\{m \in \mathbb{M}_{d}: b_{m}=b\right\}$.

In order to evaluate the adaptive scheduling schemes, the first step is to assess whether the prioritised speech calls experience an outage for the minimum HS-DSCH assignment of $p_{\mathrm{HS}-\mathrm{DSCH}}^{\mathrm{min}}$, i.e. whether speech transmission powers $\mathbf{p}_{s} \equiv\left(p_{m}, m \in \mathbb{M}_{s}\right)$ exist such that all speech calls meet their $C / I$ target,

$$
\frac{p_{m} \mathcal{G}_{m b_{m}}}{I_{s}(m)+p_{\mathrm{HS}-\mathrm{DSCH}}^{\min } I_{\boldsymbol{d}}^{\min }(m)+\nu} \geq \gamma_{\text {speech }}, m \in \mathbb{M}_{s}
$$

with $I_{s}(m) \equiv \sum_{m^{\prime} \in \mathbf{M}_{s}} \omega\left(b_{m}, b_{m^{\prime}}\right) p_{m^{\prime}} \mathcal{G}_{m b_{m^{\prime}}}\left(I_{d}^{\min }(m) \equiv\right.$ $\left.\sum_{b \in \mathbf{B}: M_{d}^{b}>0} \omega\left(b_{m}, b\right) \mathcal{G}_{m b}\right)$ the amount of interference call $m$ experiences from speech (data) transmissions (per exerted Watt of $\left.p_{\mathrm{HS}-\mathrm{DSCH}}^{\min }\right)$, and $\omega\left(b, b^{\prime}\right) \equiv \omega(1)$ if $b=b^{\prime}\left(b \neq b^{\prime}\right)$ is the applicable orthogonality factor. An additional restriction is given by the BTSs' power budget,

$$
\sum_{m \in \mathbb{M}_{a}^{b}} p_{m}+p_{\mathrm{HS}-\mathrm{DSCH}}^{\min } 1\left\{\mathbb{M}_{d}^{b} \neq \emptyset\right\} \leq p_{\max }, b \in \mathbb{B} .
$$


A distributed implementable power control scheme similar to that proposed in e.g. [1] can be applied to determine the speech performance under the minimum HS-DSCH assignment of $p_{\mathrm{HS}-\mathrm{DSCH}}^{\min }$. This implicitly determines the speech performance in terms of the number of outages.

Only if no speech outages occur in the considered constellation for the minimum HS-DSCH assignment of $p_{\mathrm{HS}-\mathrm{DSCH}}^{\mathrm{min}}$, or in case of a fixed scheduling scheme the next step is to optimise the adaptivity parameter $\vartheta_{\mathrm{HS}-\mathrm{DSCH}}$ under the condition that no speech outages are induced. Otherwise, $\vartheta_{\text {HS-DSCH }}$ is fixed at 0 .

Theorem If in a given constellation the minimum $\mathrm{HS}-\mathrm{DSCH}$ power assignment of $p_{\mathrm{HS}-\mathrm{DSCH}}^{\min }$ induces no speech outages, the optimal value of $\vartheta_{\text {HS-DSCH }}$ is given by

$$
\vartheta_{\mathrm{HS}-\mathrm{DSCH}}^{\star} \equiv \min _{b \in \mathbf{B}}\left\{\frac{\left(\begin{array}{c}
p_{\max }-p_{\mathrm{HS}-\mathrm{DSCH}}^{\min } 1\left\{\mathbb{M}_{d}^{b} \neq \emptyset\right\}+ \\
-\boldsymbol{\zeta}_{b}^{\tau}(\mathcal{I}-\mathcal{H})^{-1}\left\{p_{\mathrm{HS}-\mathrm{DSClH}}^{\min } \widehat{\mathbf{I}}_{d}^{\min }+\widehat{\nu}\right\}
\end{array}\right)}{\boldsymbol{\zeta}_{b}^{\tau}(\mathcal{I}-\mathcal{H})^{-1} \widehat{\mathbf{I}}_{d}^{\vartheta}+\varphi_{b}}\right\}
$$

where the entries of matrix $\mathcal{H}$ are given by

$$
\mathcal{H}_{m m^{\prime}} \equiv\left\{\begin{array}{cc}
0 & m=m^{\prime} \\
\frac{\omega\left(b_{m}, b_{m^{\prime}}\right) \mathcal{G}_{m b_{m}}}{\left(\gamma_{s p \operatorname{sech}}^{-1}-\omega\right) \mathcal{G}_{m b_{m}}} & m \neq m^{\prime}
\end{array},\right.
$$

for $m, m^{\prime} \in \mathbb{M}_{s}$

$$
\begin{aligned}
\widehat{\mathbf{I}}_{d}^{\text {min }} & \equiv\left(\frac{I_{d}^{\min }(m)}{\left(\gamma_{\text {speech }}^{-1}-\omega\right) \mathcal{G}_{m b_{m}}}, m \in \mathbb{M}_{s}\right), \\
\widehat{\mathbf{I}}_{d}^{\vartheta} & \equiv\left(\frac{I_{d}^{\vartheta}(m)}{\left(\gamma_{\text {speech }}^{-1}-\omega\right) \mathcal{G}_{m b_{m}}}, m \in \mathbb{M}_{s}\right), \\
\hat{\nu} & \equiv\left(\frac{\nu}{\left(\gamma_{\text {specch }}^{-1}-\omega\right) \mathcal{G}_{m b_{m}}}, m \in \mathbb{M}_{s}\right),
\end{aligned}
$$

where $I_{d}^{\vartheta}(m) \equiv \sum_{b \in \mathbf{B}} \omega\left(b_{m}, b\right) \varphi_{b} \mathcal{G}_{m b}$, and $\zeta_{b} \equiv$ $\left(1\left\{b_{m}=b\right\}, m \in \mathbb{M}_{s}\right)$ indicates which speech calls are served by BTS $b \in \mathbb{B}$.

\section{Proof See appendix.}

At this point, all HS-DSCH powers are set according to Eq. (2). In order to determine the data QOS, the distinction in microscheduling schemes (RR or WRR) needs to be effectuated. Recall that since data flows inherently meet their $C / I$ targets via
HSDPA's link adaptation feature, data flow $m \in \mathbb{M}_{d l}$ achieves a throughput of (cf. Eq.

(1))

$R_{m}^{\prime}=\frac{R_{\text {chip }}}{10 \zeta_{\text {dat }} / 10} \frac{p_{\mathrm{HS}-\mathrm{DSCH}}^{b_{m}} \mathcal{G}_{m b_{m}}}{I_{s}(m)+p_{\mathrm{HS}-\mathrm{DSCH}}^{\min } I_{d}^{\min }(m)+\vartheta_{\mathrm{HS}-\mathrm{DSCH}} I_{d}^{\vartheta}(m)+\nu}$

kbits/s whenever it is served, so that its expected throughput during the scheduling period is given by $R_{m}=\phi_{m}^{b_{m}} R_{m}^{\prime}$, where the micro-scheduling weights $\phi_{m}^{b_{n}}$ depend on the deployed microscheduling alternative:

$$
\begin{gathered}
\phi_{m}^{b_{m}}= \begin{cases}1 / M_{d}^{b_{m}} & (\mathrm{RR}) \\
\frac{1 / R_{m}^{\prime}}{\sum_{m / \in M_{d}^{b_{m}}} 1 / R_{m \prime}^{\prime}} & \text { (WRR) }\end{cases} \\
\text { V. NUMERICAL RESULTS }
\end{gathered}
$$

A Monte Carlo simulation study is carried out to evaluate the scheduling schemes with respect to the speech and data performance. $M_{s}$ and $M_{d}$ are assumed to be Poisson random variables with mean $\rho_{\text {speech }}$ and $\rho_{\text {data }}$, respectively.

A single cell scenario is considered. The system and traffic parameters are given by $R=1 / \sqrt{3} \mathrm{~km}, p_{\max }=42 \mathrm{dBm}(\approx$ 15.849 Watt $), \nu=-99.157 \mathrm{dBm}\left(\approx 1.21410^{-13}\right.$ Watt $), \varsigma=$ $3.523, \sigma=8 \mathrm{~dB}, \omega=0.40, R_{\text {chip }}=3.84 \mathrm{Mchips} / \mathrm{s}, R_{\text {speech }}=$ $12.2 \mathrm{kbits} / \mathrm{s}, \zeta_{\text {speech }}=5 \mathrm{~dB}$ and $\zeta_{\text {data }}=4 \mathrm{~dB}$.

For $\left(\rho_{\text {speech }}, \rho_{\text {data }}\right)=(75,25)$ Fig. 2 depicts the speech and data performance as a function of $p_{\mathrm{HS} \cdot \mathrm{mSCH}}^{\min }$ for both the fixed and adaptive macro-scheduling schemes, and for the RR (left) and WRR (right) micro-scheduling alternatives. The vertical bars along the $\mathbf{R}_{d}$ curves indicate the standard deviation of the data throughput. Observe first the trivial trends that both $\mathbf{P}_{s}$ and $\mathbf{R}_{d}$ are increasing in $p_{\mathrm{HS}-\mathrm{DSCH}}^{\min }$, and that $\mathbf{P}_{s}$ is inherently insensitive to both the scheme's adaptivity at the macro-level and the choice of micro-scheduling scheme. With regard to $\mathbf{R}_{d}$, the adaptive scheme appears to exploit its minimum guarantee of $p_{\mathrm{HS}-\mathrm{DSCH}}^{\mathrm{min}}$ not until it exceeds about 5 Watt, beyond which the adaptivity gain vanishes as both throughputs curves converge. Assessing the impact of the micro-scheduling scheme, observe that the 'rate-fair' WRR scheme yields lower expected throughputs. This can be intuitivitly explained by the insight that the WRR scheme assigns a disproportionate amount of resources to the data flow at the worst location, thereby reducing the overall data performance. In contrast, under the 'power fair' RR scheme such a badly located data flow receives a fair share of the resources, and thus does not inflict its own suffering upon the other data flows. 

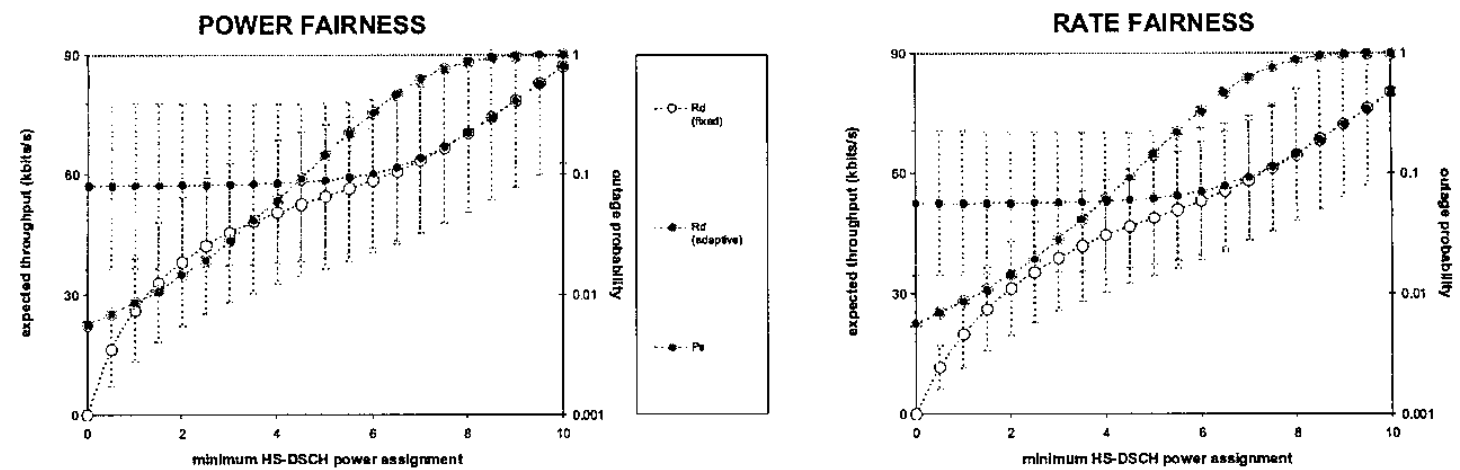

Fig. 2. Speech and data performance versus $\rho_{\mathrm{HSOSCH}}^{\min }\left(\rho_{\text {speech }}=75\right.$, $\left.\rho_{\text {data }}=25\right)$.
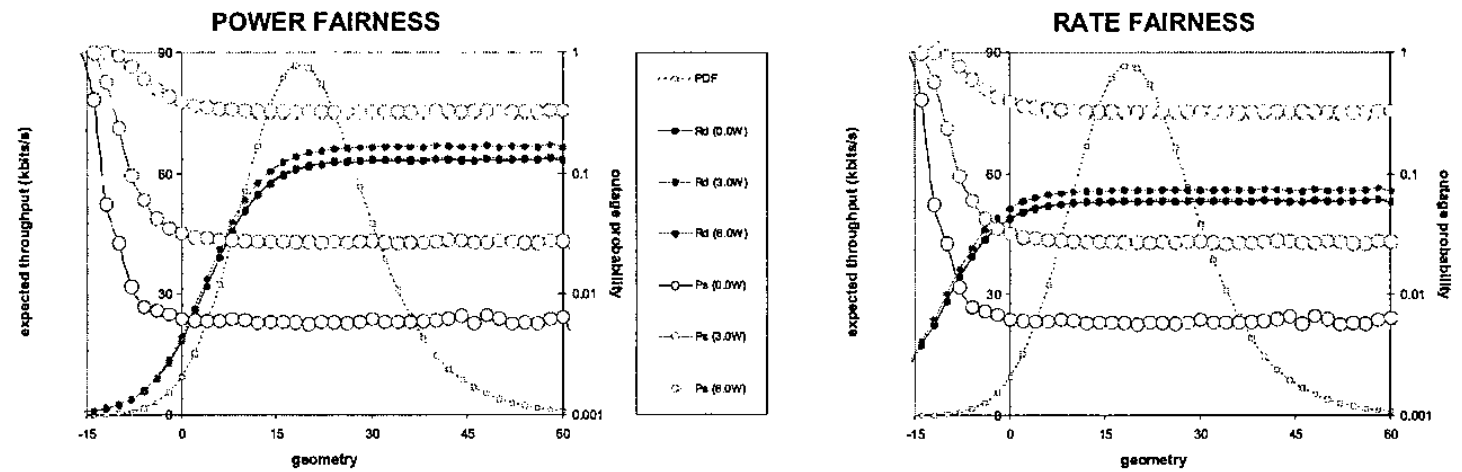

Fig. 3. Conditional speech and data performance for different $\mathrm{p}_{\mathrm{HS}-\mathrm{mSCH}}^{\min }\left(\rho_{\text {speach }}=75, \rho_{\text {data }}=25\right)$.
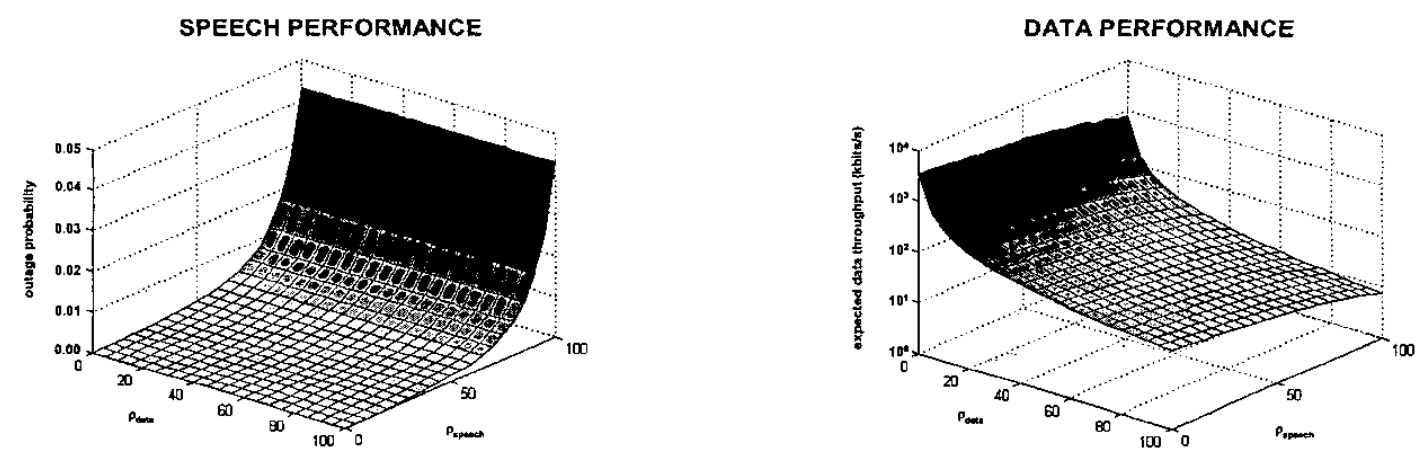

Fig. 4. Speech and data performance versus $\left(\rho_{\text {speech }}, \rho_{\text {data }}\right)$. 
Fig. 2 further demonstrates that a purely adaptive scheme can improve both speech and data performance jointly, compared to a scheme with fixed HS-DSCH powers.

Fig. 3 shows the conditional speech and data performance under the adaptive scheduling scheme with $p_{\mathrm{HS}-\mathrm{DSCH}}^{\min } \in\{0,3,6\}$ Watt, and for the RR (left) and WRR (right) micro-scheduling alternative. As expected, the experienced speech and data performance improve as a terminal is located nearer to the BTS (higher geometry). The principal result demonstrated by the figure is the impact of the micro-scheduling scheme: due to the 'rate fairness' of the WRR scheme, distant terminals typically experience higher throughputs than under the RR scheme, which favours near terminals. In this single cell case, the RR scheme outperforms the WRR scheme w.r.t. to both the expected throughput and the conditional expected throughput experienced at the modal geometry.

Fig. 4 shows $\mathbf{P}_{s}$ and $\mathbf{R}_{d}$ under the purely adaptive scheduling scheme ( $\left.p_{\mathrm{HS}-\mathrm{DSCH}}^{\min }=0 \mathrm{Watt}\right)$ with RR micro-scheduling as a function of the traffic mix $\left(\rho_{\text {speech }}, \rho_{\text {data }}\right)$. Aside from the inherent insensitivity of $\mathbf{P}_{\boldsymbol{s}}$ to $\rho_{\text {data }}$ under the purely adaptive scheduling scheme, performance generally worsens under heavier traffic. Observe that whereas $\mathbf{P}_{\boldsymbol{s}}$ increases exponentially in $\rho_{\text {speech }}$, $\mathbf{R}_{d}$ becomes less and less sensitive as the traffic load increases. For light traffic loads $\mathbf{R}_{d}$ is most sensitive to $\rho_{\text {data }}$, while for relatively heavy traffic loads the relative impact of $\rho_{\text {speech }}$ becomes slightly dominant. In case of WRR micro-scheduling, the $\mathbf{R}_{d}$ curve lies below that for the RR scheme (not shown), with the discrepancy increasing in $\rho_{\text {data }}$ due to the growing significance of the badly located terminals.

\section{CONCLUDING REMARKS}

A performance evaluation of adaptive scheduling schemes has been presented for the downlink in a UMTS network integrating prioritised speech and delay-tolerant data calls. We have derived a closed-form expression for the optimal transmission power assigned to the HS-DSCHS that are applied to multiplex the data flows, while protecting the speech calls' $C / I$ performance. Using a fixed $\mathrm{HS}-\mathrm{DSCH}$ power assignment as a reference scheme, the numerical experiments have demonstrated that it it possible to improve both the speech and data performance by means of adaptive scheduling. A minimum HS-DSCH power assignment was demonstrated to be an effective policy parameter to attain a desired tradeoff between speech and data performance.

For further research we recommend to apply the derived analytical results in dynamic simulations (see also [4]) in order to further investigate the implications of the demonstrated spatial unfaimess as the system evolves in time, and to devise heuristic derivations of the optimal scheme for practical implementation.

\section{ACKNOWLEDGMENTS}

The authors appreciate the valuable comments of Richard $\mathbf{J}$. Boucherie of the University of Twente, The Netherlands. This research has been carried out within the BEYOND 3G project, and is partially supported by the Ministry of Economic Affairs, The Netherlands.

\section{APPENDIX: PROOF OF THEOREM}

Proof Along similar lines as followed in e.g. [1], the speech calls' $C / I$ conditions for a given value of $\vartheta_{\mathrm{HS}-\mathrm{DSCH}}$ can be written in matrix form:

$$
(\mathcal{I}-\mathcal{H}) \mathbf{p}_{s} \geq p_{\mathrm{HS}-\mathrm{DSCH}}^{\min } \widehat{\mathbf{I}}_{d}^{\min }+\vartheta_{\mathrm{HI}-\mathrm{DSCH}} \widehat{\mathbf{I}}_{d}^{\vartheta}+\widehat{\nu}
$$

Momentarily ignoring the restrictions posed by the BTS power budget $p_{\max }$, the Pareto optimal vector of speech transmission powers is given by

$$
\mathbf{p}_{s}^{\star} \equiv(\mathcal{I}-\mathcal{H})^{-1}\left\{p_{\mathrm{HS}-\mathrm{DSCH}}^{\min } \widehat{\mathbf{I}}_{d}^{\min }+\vartheta_{\mathrm{HS}-\mathrm{DSCH}} \widehat{\mathbf{I}}_{d}^{\vartheta}+\widehat{\nu}\right\}
$$

which is nonnegative and increasing in the aggregate adaptive HS-DSCH power component $\vartheta_{\text {HS-DSCH }} \geq 0$, since the matrix $(\mathcal{I}-\mathcal{H})^{-1}$ is positive componentwise [1]. A necessary and sufficient restriction for $\vartheta_{\mathrm{HS}-\mathrm{DSCH}}$ from BTS $b$ 's perspective is then

$$
\zeta_{b}^{\tau} \mathbf{P}_{s}^{\star}+p_{\mathrm{HS}-\mathrm{DSCH}} 1\left\{\mathbb{M}_{d}^{b} \neq \emptyset\right\}+\varphi_{b} \vartheta_{\mathrm{HS}-\mathrm{DSCH}} \leq p_{\max },
$$

for each $b \in \mathbb{B}$. Since these restrictions on $\vartheta_{\mathrm{HS}-\mathrm{DSCH}}$ must hold for all BTSs, Eq. (3) immediately follows.

\section{REFERENCES}

[1] N.D. Bambos, S.C. Chen and G.J. Pottie, "Radio link admission algorithms for wireless networks with power control and active link quality protection," Proceedings of IEEE INFOCOM '95, Boston, USA, 1995.

[2] S.C. Borst and P. Whiting, "Dynamic rate control algorithms for HDR throughput optimisation," Proceedings of IEEE INFOCOM '01, Anchorage, USA, 2001.

[3] R. DeBernardi, D. Imbeni, L. Vignali and M. Kerlsson, "Load control strategies for mixed services in WCDMA," Proceedings of IEEE VTC '00, Tokyo, Japan, 2000.

[4] V.M. Espinosa Velez, L. Jorguseski, R. Litjens, E.R. Fledderus and R. Prasad, "Downlink Radio Resource Estimation and Control in WCDMA Cellular System with Voice and Data Users," Proceedings of IEEE WPMC '01, Aalborg, Denmark, 2001.

[5] H. Holma and A. Toskala, "WCDMA for UMTS: radio access for third generation mobile communications," John Wiley \& Sons, Chichester, England, 2001.

[6] J.M. Holtzman, "CDMA forward link waterfilling power control," Proceedings of IEEE VTC '00, Tokyo, Japan, 2000.

[7] I. López, P.J. Ameigeiras, J. Wigard and P. Mogensen, "Downlink radio resource management for IP packet services in UMTS," Proceedings of IEEE VTC '01, Rhodes, Greece, 2001.

[8] S. Parkvall, E. Dahlman, P. Frenger, P. Beming and M. Persson, "The evolution of WCDMA towards higher speed downlink packet data access," Proceedings of IEEE VTC '01, Rhodes, Greece, 2001.

[9] J. Sachs, T. Balon and M. Meyer, "Congestion control in WCDMA with respect to different service classes," Proceedings of European Wireless '99, Munich, Germany, 1999.

[10] A.J. Viterbi, "CDMA: principles of spread spectrum communication," Addison-Wesley, Reading, USA, 1995. 